\title{
Adaptive inelastic magnetic mirror for Bose-Einstein condensates
}

\author{
A. S. Arnold, C. MacCormick, and M. G. Boshier \\ Sussex Centre for Optical and Atomic Physics, University of Sussex, Brighton BN1 9QH, United Kingdom
}

(Received 28 March 2001; published 30 January 2002)

\begin{abstract}
We report the reflection and focusing of a Bose-Einstein condensate by a pulsed magnetic mirror. The mirror is adaptive, inelastic, and has extremely high optical quality. Deviations from specularity are below $1 \mathrm{mrad} \mathrm{rms}$ — almost an order of magnitude better than other atomic mirrors. The mirror has been used to realize the atom-optical analog of a beam expander, producing an ultracold collimated fountain of matter waves. The results of these experiments are in good agreement with simple theoretical models.
\end{abstract}

DOI: 10.1103/PhysRevA.65.0216XX

PACS number(s): 03.75.Fi, 03.75.Be, 32.80.-t, 05.60.Gg

A major aim of the field of atom optics is to build analogs of mirrors, lenses, and waveguides for manipulating cold atoms and matter waves in applications ranging from highresolution lithography to ultrasensitive atom interferometry [1]. Almost all prior experiments in this area have used conventional laser-cooled atoms: cold atomic clouds have been reflected and focused by magnetic fields and the optical dipole potential [2-8], and cold atoms have been focused in both one [9] and three [10] dimensions using pulsed magnetic fields. However, since a gaseous Bose-Einstein condensate (BEC) [11] is the ultimate source of coherent atoms, it is extremely important to develop high-quality atom-optical elements that can be used to manipulate BECs. In spite of the intense theoretical interest in matter wave optics, the first experimental step in this direction came only recently, with the demonstration of a flat optical dipole force mirror [12].

In this paper we show that a different kind of atomic mirror [13], based on pulsed magnetic fields [13,14], can be used to reflect and focus Bose condensates, and we demonstrate that it has substantially higher optical quality than any other atomic mirror. The mirror is also inelastic and it can produce adjustable three-dimensional (3D) focusing. These attractive features allow us to realize a BEC "beam expander," which produces an extremely cold, collimated beam of matter waves. Finally, we show that the evolution of a BEC bouncing on the mirror is in good agreement with theory, and discuss the limitations of the most widely used theory of BEC expansion.

All magnetic atom-optical elements make use of the Stern-Gerlach potential $U=-\mu B$ experienced by an atom moving adiabatically in a magnetic field of magnitude $B$ (with $\mu$ being the component of the atomic magnetic moment parallel to the field). For weak-field seeking atoms, any field for which $B$ increases in the direction of the initial atomic velocity will act as a simple mirror. If the atoms are to be focused as well as reflected, then $B$ must in addition have positive curvature in the appropriate directions. Other desirable properties of a condensate mirror are equipotential surfaces sufficiently smooth to preserve coherence, and compatibility with the ultrahigh-vacuum environment of a typical BEC apparatus. Since these conditions must all be satisfied by the magnetic trap in which the condensate is formed, the trap field itself provides an ideal starting point for the construction of a mirror. It is particularly important here that all magnetic elements in the trap are a relatively large distance $d \gtrsim 20 \mathrm{~mm}$ from the atoms. Thus, as curvature in the magnetic field scales as $d^{-3}$, microscopic corrugations are drastically reduced compared to mirrors in which atoms make a close $(d<100 \mu \mathrm{m})$ approach to the surface. Such corrugations, and their optical analogs, presently limit the quality of existing atomic mirrors $[3,4,8,15]$ and so large-scale mirrors might provide an easier route to fully coherent atomic manipulation. Also, our mirror focuses the BEC in 3D, at least to some extent, with a single magnetic pulse, in contrast to previous work with cold atoms which has either been restricted to 1D [9] or required the judicious application of two pulses [10].

In our experiment the magnetic mirror is formed by combining a horizontal Ioffe-Pritchard [16] magnetic trap with a uniform vertical magnetic field $B_{c}$. We use a coordinate system where the symmetry axis of the trap is in the $z$ direction, gravity acts in the $-y$ direction, and the origin is at the center of the magnetic trap. The total magnetic mirror field to second order is then

$$
\begin{aligned}
\mathbf{B}(\mathbf{r})= & \left\{0, B_{c}, B_{0}\right\}+B^{\prime}\{x,-y, 0\}+B^{\prime \prime} / 2\left\{-x z,-y z, z^{2}\right. \\
& \left.-\left(x^{2}+y^{2}\right) / 2\right\} .
\end{aligned}
$$

The parameters $B_{0}, B^{\prime}$, and $B^{\prime \prime}$ are, respectively, the bias field, gradient, and curvature of the trap. One can show that the magnitude of this field (Fig. 1) is essentially parabolic in the axial $(z)$ direction, but hyperbolic in the offset radial coordinate $r=\sqrt{x^{2}+\left(y-y_{c}\right)^{2}}$ and thus effectively linear for radii $r>\sqrt{2} B_{0} / B^{\prime}$. The potential closely approximates that of a horizontal cylindrical mirror. The control field $B_{c}$ allows us to shift the minimum of the potential vertically from $y$

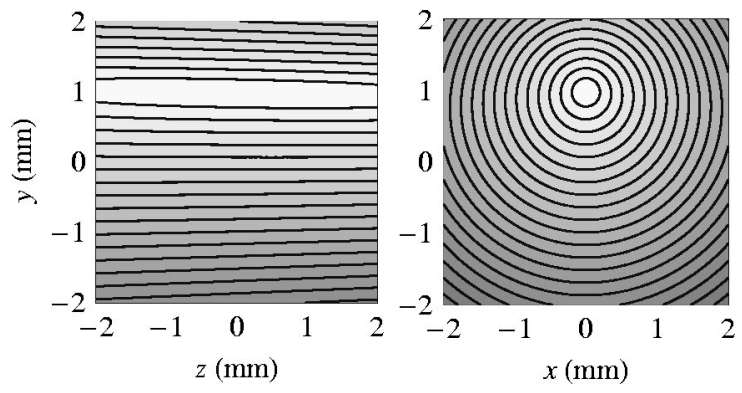

FIG. 1. Contours of the total magnetic mirror and gravitational potential for the case $y_{c}=1 \mathrm{~mm}$. 


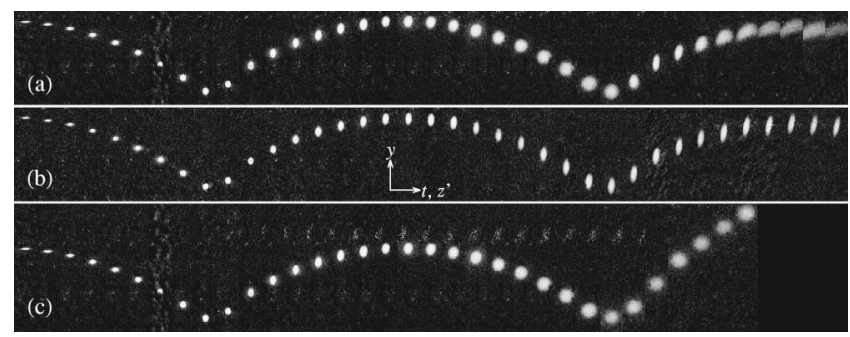

FIG. 2. Sequences of experimental images taken at times $t$ $=2,4, \ldots, 74 \mathrm{~s}$ for control fields (a) $B_{c}=0$ and (b) $B_{c}=70 \mathrm{G}$. Each image in a sequence is $0.5 \mathrm{~mm}$ wide (the $z^{\prime}$ direction) and 2.0 $\mathrm{mm}$ high (the $y$ direction). For sequence (c) the control field is $B_{c}$ $=0$ during the first bounce, and the control field and pulse duration during the second bounce are chosen to collimate and launch the BEC. The individual images in (c) have dimensions 0.5 $\times 2.7 \mathrm{~mm}^{2}$.

$=0$ to $y_{c}=B_{c} / B^{\prime}$, moving the center of curvature of the mirror to $y_{c}$ and making the radius of curvature at a particular height $-y$ below the trap center equal to $y+y_{c}$. A condensate falling under gravity can be reflected by a brief pulse of the magnetic mirror field. During the pulse the atoms experience a potential that is weakly confining in the $z$ direction, strongly confining (with strength dependent on $B_{c}$ ) in the $x$ direction, and almost linear in the $y$ coordinate. Linear variation of the potential with height results simply in a change in center-of-mass motion (i.e., bouncing), whereas the parabolic dependence in the two horizontal directions focuses the condensate. Field pulses repeated at appropriate intervals produce multiple reflections.

The BECs [17] are produced by the route most commonly used to date: laser cooling, magnetic trapping, and evaporative cooling. The magnetic trap has a bias field of $1 \mathrm{G}$, with measured axial and radial trapping frequencies of $\nu_{z}$ $=10 \mathrm{~Hz}$ and $\nu_{r}=223 \mathrm{~Hz}$ at a current of $I_{\text {trap }}=220 \mathrm{~A}$. Pure condensates are reproducibly obtained with more than $10^{5}\left|F, m_{F}\right\rangle=|2,2\rangle$ atoms. The atoms are imaged in absorption using an $8 \mu$ s pulse of resonant light from a beam which propagates horizontally at an angle of $60^{\circ}$ to the $z$ axis of the magnetic trap. We refer to the resulting horizontal axis in our images as $z^{\prime}$. The imaging system has a magnification of $0.80(1)$.

Figures 2(a) and 2(b) are sequences showing reflection and focusing of the condensate by mirrors of small and large radii of curvature, respectively. The mirror was pulsed on with $I_{\text {trap }}=155$ A for $5 \mathrm{~ms}$ every $35 \mathrm{~ms}$. This pulse duration gives an elastic bounce for our magnetic acceleration $\mu B^{\prime} / m \approx 6 g$. In these sequences we have suppressed a horizontal motion of the condensate, discussed below, to illustrate the bouncing more clearly and to focus attention on the evolution of the condensate shape.

In Fig. 2(a) the BEC width grows rapidly because in this case the cavity formed by the magnetic mirror and gravity is unstable, meaning that the classical trajectories walk out of the cavity. Gravity cavities are unstable when the radius of curvature $R$ of the mirror is less than twice the release height $h[8,19,20]$, and in Fig. 2(a) the radius of curvature in the $x y$ plane $R_{x y}=h \approx 1.4 \mathrm{~mm}$. The classical motion in this case is easily visualized. Since the gravitational potential energy is much larger than the initial kinetic energy of the BEC, all trajectories strike the mirror at an angle very close to vertical. The reflected trajectories then cross the mirror axis at a focal point $\approx 0.5 R_{x y}$ above the mirror surface, on their way to turning points at height $h$. The BEC will be strongly focused through this focal point after each bounce, as can be seen after the second bounce in Fig. 2(a), even though our viewpoint is at an angle of $60^{\circ}$ to the $z$ axis. In contrast, Fig. 2(b) corresponds to the stable case $R_{x y}=5 h$.

A complete theoretical analysis of our experiment would require a numerical integration of the Gross-Pitaevskii equation on a large mesh in $3+1$ dimensions, a rather formidable task. We have therefore developed two simpler theoretical models of the bounce dynamics. The first extends the widely used result [18] that, in the Thomas-Fermi regime, a BEC confined in a time-dependent parabolic potential $U(\mathbf{r}, t)$ $=(m / 2) \sum_{j=1}^{3} \omega_{j}(t)^{2} r_{j}^{2}$ has an atomic spatial distribution that obeys the simple scaling law

$$
n(\mathbf{r}, t)=\max \left\{\frac{n_{0}}{\lambda_{1} \lambda_{2} \lambda_{3}}\left(1-\sum_{j=1}^{3} \frac{r_{j}^{2}}{A_{j}^{2} \lambda_{j}^{2}}\right), 0\right\}
$$

where $\lambda_{j}(t)$ is the solution of

$$
\frac{d^{2} \lambda_{j}(t)}{d t^{2}}+\omega_{j}(t)^{2} \lambda_{j}(t)-\frac{\omega_{j}(0)^{2}}{\prod_{i=1}^{3} \lambda_{i}(t)^{1+\delta_{i j}}}=0,
$$

$\lambda_{j}(0)=1$, and the initial Thomas-Fermi radii are $A_{j}$. We extend this with the result [17] that if the potential is parabolic about the time-varying center $\mathbf{r}_{c}(t)$ of the BEC, i.e., if $U(\mathbf{r}, t)=(m / 2) \sum_{j=1}^{3} \omega_{j}(t)^{2}\left[r_{j}-r_{c j}(t)\right]^{2}$, then the same scaling law applies for the BEC if one uses the magnetic curvatures acting at the condensate center. This model is very fast to compute, and includes the effects of mean-field repulsion. In order to verify that this locally harmonic potential approximation is valid for our mirror, we developed a second model, based on a Monte Carlo simulation. This model follows the classical trajectories of $10^{5}$ atoms with initial positions and velocities randomly chosen to give the ThomasFermi result for the free expansion [Eq. (2)]. In this model the mirror potential can be treated exactly, but atomic interactions after the initial mean-field energy driven expansion are neglected. Both theoretical models make the reasonable approximation that diffraction is negligible, and both use measured values of the parameters characterizing our mirror $\left(B_{0}, B^{\prime}, B^{\prime \prime}, B_{c}\right)$. To keep the computing time manageable, the simulations use the magnetic field given by Eq. (1) with an extra term added to account for the second-order curvature in our control field. The acceleration produced by this approximate field differs from the results of an accurate numerical calculation for our coil geometry by less than $0.1 \%$ over the $\sim 1.5 \mathrm{~mm}$ radius region explored by the condensate in the experiments reported here.

Figure 3 shows that the Monte Carlo simulation is in excellent agreement with the experimental data of Fig. 2(b). The horizontal motion seen here (and suppressed in Fig. 2) 


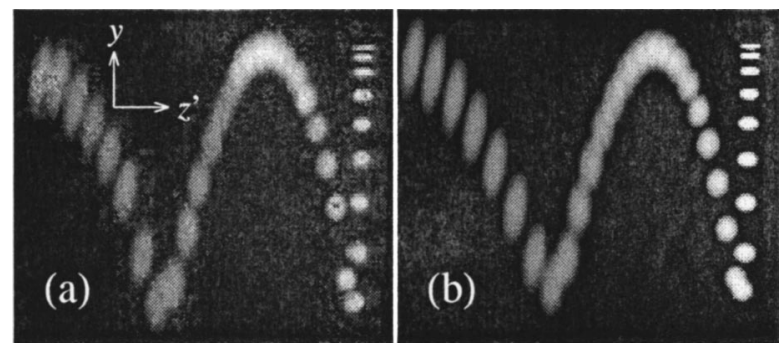

FIG. 3. (a) Superimposed experimental images for times $t$ $=2,4, \ldots, 68 \mathrm{~ms}$ showing the full center-of-mass motion for a mirror with $B_{c}=70 \mathrm{G}$ [i.e., Fig. 2(b)]. The image is in the $z^{\prime} y$ plane, with size $2.2 \times 1.9 \mathrm{~mm}^{2}$. (b) Monte Carlo simulation of the condensate evolution for the same conditions.

arises because of a small tilt of the magnetic trap and because the magnetic potential has an increasingly large slope in the $z$ direction as $y$ decreases (Fig. 1). This slope also causes the condensate to rotate in the $y z$ plane during a bounce. The Thomas-Fermi calculation yields very similar results, except the rotation of the condensate is not reproduced because that model assumes a perfectly harmonic trap. The horizontal motion limited the number of bounces that we could observe to two, which was sufficient for characterizing the mirror. However, the horizontal motion could, if necessary, be suppressed in future experiments. Since one finds from Eq. (1) that the slope in the potential at a given height $y$ is almost independent of $B_{c}$, the slope could be compensated at a particular bouncing height by simply tilting the coils slightly. A better, although more complex, solution is rotating the magnetic trap by $45^{\circ}$ about the trap axis and applying the control field in the $x$ direction, because in this geometry the potential surfaces at all positions below the trap center are horizontal for all values of $B_{c}$.

To compare the experimental data with these models quantitatively, we obtained condensate radii and rotation angles $\theta$ (measured as shown in the inset to Fig. 4) by fitting the absorption images to a Thomas-Fermi column density. The extended Thomas-Fermi model gives these radii directly. For the Monte Carlo model, the radii are deduced from the moments $\langle y\rangle,\left\langle z^{\prime}\right\rangle,\left\langle y^{2}\right\rangle,\left\langle z^{\prime 2}\right\rangle$, and $\left\langle y z^{\prime}\right\rangle$. Figure 4 shows that the two models are both in good agreement with each other and with the experimental data, confirming the validity of their respective approximations. The fitted values of $\theta$ are in reasonable agreement with the predictions of the Monte Carlo model.

The origin of the very small difference between the two models was found by selectively suppressing possible mechanisms in the calculations. Removing the densitydependent term in the Thomas-Fermi calculation just before the first bounce has a negligible effect, confirming that interactions are not important after the initial ballistic expansion. However, repeating the Monte Carlo simulation with the harmonic potential used in the Thomas-Fermi calculation brings the two models into complete agreement, indicating that the difference between the models is due to nonparabolic terms in the mirror potential. Further numerical experiments showed that the second-order term proportional to $y z$ is mostly responsible. Although here the difference is small, it

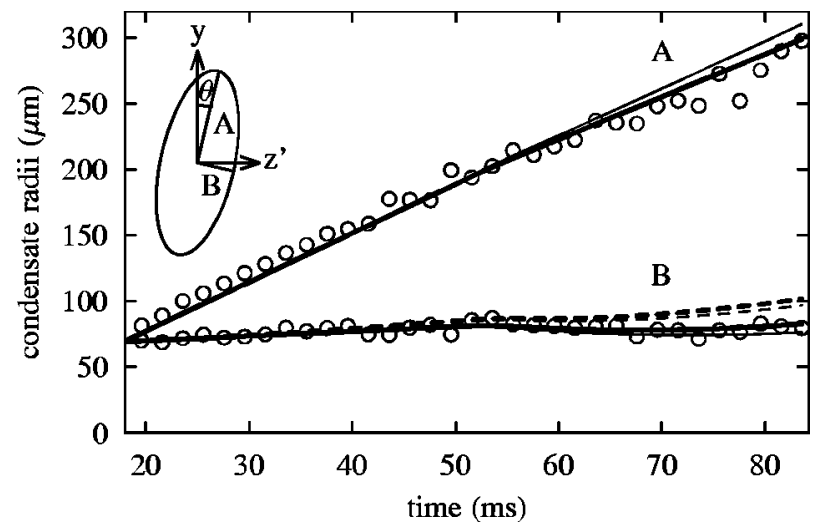

FIG. 4. Circles show the experimentally determined BEC radii as a function of time for a condensate bouncing on a mirror with $R_{x y}=5 h$ [Fig. 2(b)]. The light and bold curves show the predictions of the Monte Carlo and Thomas-Fermi models, respectively. The two dashed curves show the corresponding predictions for the case of a mirror with rms roughness of $1 \mathrm{mrad}$. The total experimental error due to exposure time, resolution, finite pixel size, and lensing [21] is $\lesssim 2 \mu \mathrm{m}$ for radii $R>60 \mu \mathrm{m}$, which is negligible compared to the shot-to-shot statistical fluctuations.

will be important for modeling bouncing BECs over longer times, and in these cases the extended Thomas-Fermi model may not be sufficiently accurate.

Since the BEC dynamics are well described by the two models, they can be used to quantify the optical quality of the mirror. The horizontal size of the bouncing condensate is a sensitive probe of mirror quality because any imperfections will increase this dimension of the condensate on each bounce. We therefore modified the two simulations to add the effects of a $1 \mathrm{mrad}$ rms mirror roughness to the condensate on each bounce. The results are shown as dashed curves in Fig. 4. The substantial discrepancy between these curves and the experimental data implies that any deviations from specularity in the mirror are considerably less than $1 \mathrm{mrad}$. This performance is better than the best published atomic mirror data $[3,4,15]$ by a factor of $5-6$, making this pulsed magnetic mirror the smoothest cold atom mirror to date. The upper bound on roughness might be improved in future experiments by simply extending the observation time.

In addition to extremely high optical quality, the mirror also has the useful properties of being adaptive (changing $B_{c}$ changes the focal length) and inelastic (changing the pulse duration changes the vertical impulse imparted to the BEC). A second experiment was performed to explicitly take advantage of these aspects of the mirror. We realized a BEC "beam expander," by first expanding the BEC by reflection from a short radius $\left(R_{x y}=h\right)$ mirror, and then on the second reflection launching [22] and collimating it by a longer interaction with a weakly focusing mirror. The results are shown in Fig. 2(c). This process produces a low-density, ultracold, condensate fountain, which might, e.g., be useful in atomic clocks. It can also be regarded as a demonstration of $\delta$-function-kick cooling [23].

Figure 4 shows that the evolution of a condensate reflected by the magnetic mirror is well described by classical physics. This is in contrast to the reflection of condensates by 
light sheets [12], where the reflected condensate develops a complicated self-interference structure near the turning point of its trajectory. Fringes occur when the de Broglie wavelength of the condensate is large at positions where the classical atomic trajectories cross, a condition that is never satisfied for our mirror. Of course, in many atom-optical applications the absence of interference fringes is an advantage.

Our two theoretical models do suggest that nonclassical evolution should be visible in the $x y$ plane under certain conditions. In particular, for the case $R_{x y}=h$ [Fig. 2(a)] the density and the "waist" size at the tight focus near the $R_{x y} / 2$ point are respectively high enough and small enough that the effects of atomic interactions and diffraction might be visible. The condensate's behavior near this tight waist provides an extremely sensitive probe of the mirror quality, making it interesting to image the condensate along the trap axis and compare the results with a calculation using the timedependent 3D Gross-Pitaevskii equation. Another topic for future investigation is the coherence of the mirror. Since the trap field is sufficiently well controlled to produce a condensate in the first place, it seems very likely that the mirror will preserve coherence. Nevertheless, it would be desirable to demonstrate this directly, e.g., by splitting a condensate coherently and interfering the two reflections.

In conclusion, we have demonstrated adjustable focusing and reflection of a BEC from a pulsed magnetic mirror, and shown that the evolution of the reflected condensate is well described by simple theories. The mirror sets a limit better by almost an order of magnitude for the optical quality of atom-optical elements. Finally, we have used the adaptive and inelastic properties of the mirror to realize an atomic "beam expander," and used it to make an ultracold matter wave fountain.

We gratefully acknowledge valuable discussions with Ed Hinds. This work was supported by the U.K. EPSRC and the University of Sussex. A.S.A. was funded by the Commonwealth Scholarship Commission.
[1] Atom Optics, edited by M.G. Prentiss and W.D. Phillips, Proceedings of the SPIE Vol. 2995 (SPIE, Bellingham, WA, 1997).

[2] T.M. Roach et al., Phys. Rev. Lett. 75, 629 (1995).

[3] C.V. Saba et al., Phys. Rev. Lett. 82, 468 (1999).

[4] M. Drndić et al., Phys. Rev. A 60, 4012 (1999).

[5] D.C. Lau et al., Eur. Phys. J. D 5, 193 (1999).

[6] M.A. Kasevich et al., Opt. Lett. 15, 607 (1990).

[7] C.G. Aminoff et al., Phys. Rev. Lett. 71, 3083 (1993).

[8] E.A. Hinds and I.G. Hughes, J. Phys. D 32, R119 (1999).

[9] E. Marechal et al., Phys. Rev. A 59, 4636 (1999).

[10] E.A. Cornell et al., Phys. Rev. Lett. 67, 2439 (1991).

[11] M.H. Anderson et al., Science 269, 198 (1995); K.B. Davis et al., Phys. Rev. Lett. 75, 3969 (1995); C.C. Bradley et al., ibid. 79, 1170 (1997).

[12] K. Bongs et al., Phys. Rev. Lett. 83, 3577 (1999).

[13] A.S. Arnold, C. MacCormick, and M.G. Boshier, in Laser Spectroscopy XIV, edited by R. Blatt et al. (World Scientific, Singapore, 1999), p. 318.
[14] T. Esslinger et al., in Bose-Einstein Condensates and Atom Lasers, edited by S. Martellucci et al. (Kluwer Academic, Dordrecht, 2000), p. 117.

[15] N. Westbrook et al., in Atomic Physics 17, edited by E. Arimondo et al. (AIP, Melville, NY, 2001), p. 397.

[16] D.E. Pritchard, Phys. Rev. Lett. 51, 1336 (1983).

[17] A.S. Arnold and M.G. Boshier (unpublished).

[18] Yu. Kagan, E.L. Surkov, and G.V. Shlyapnikov, Phys. Rev. A 54, R1753 (1996); Y. Castin and R. Dum, Phys. Rev. Lett. 77, 5315 (1996).

[19] H. Wallis et al., Appl. Phys. B: Photophys. Laser Chem. 54, 407 (1992).

[20] I.G. Hughes et al., J. Phys. B 34, 2869 (2001).

[21] M.R. Andrews et al., Science 273, 84 (1996).

[22] C.G. Townsend et al., in Atomic Physics 15, edited by H.B. van Linden van den Heuvell et al. (World Scientific, Singapore, 1997), p. 206.

[23] H. Ammann and N. Christensen, Phys. Rev. Lett. 78, 2088 (1997). 